\title{
CONSIDERAÇÕES SOBRE A CONSULTA DE ENFERMAGEM: O OLHAR DE HIPERTENSOS DA ATENÇÃO PÚBLICA PRIMÁRIA NO MUNICÍPIO DE PETROLINA/PE
}

\author{
Luciana Dantas Farias de ANDRADE ${ }^{1}$ \\ Priscila Dayane Marques CARVALHO ${ }^{2}$ \\ Maria Benegelania PINTO ${ }^{3}$ \\ Nathanielly Cistina Carvalho de Brito SANTOS ${ }^{4}$ \\ Édija Anália Rodrigues de LIMA
}

\begin{abstract}
${ }^{1}$ Enfermeira. Docente no Curso de Bacharelado em Enfermagem na Universidade Federal de Campina Grande - UFCG, campus Cuité - PB. Doutora em Psicologia. CPF: 008.951.974-48. RG: 250.4778 SSP-PB. Endereço: Rua Comerciante José Miranda de Araújo, 185/1403 - Jardim Oceania. CEP: 58037-428 - João Pessoa - PB. E-mail:

luciana.dantas.farias@gmail.com
\end{abstract}

${ }^{2}$ Enfermeira. Residência Multiprofissional em Saúde da Família (UNIVASF). Especialista em Enfermagem do trabalho. Enfermeira do trabalho da EMBRAPA. E-mail: prisciladaiane@msn.com

${ }^{3}$ Enfermeira. Docente no Curso de Bacharelado em Enfermagem na Universidade Federal de Campina Grande - UFCG, campus Cuité - PB. Mestre em Enfermagem na Atenção à Saúde. E-mail: benegelania@ yahoo.com.br

${ }^{4}$ Enfermeira. Docente no Curso de Bacharelado em Enfermagem na Universidade Federal de Campina Grande - UFCG, campus Cuité - PB. Especialização em Serviços de Saúde Pública e Educação Profissional da Área de Saúde (Enfermagem); Mestranda no Programa de Pós-Graduação na Universidade Federal da Paraíba. E-mail: nathcris@hotmail.com

${ }^{5}$ Enfermeira. Docente no Curso de Bacharelado em Enfermagem na Universidade Federal de Campina Grande - UFCG, campus Cuité - PB. Mestre em Enfermagem na Atenção à Saúde. E-mail: edijaprof@hotmail.com

Recebido em: 31/08/2013 - Aprovado em: 21/12/2013 - Disponibilizado em: 15/01/2014

RESUMO: O objetivo desse estudo foi conhecer o entendimento que os hipertensos têm acerca da consulta de enfermagem realizada pelos profissionais da área e como a mesma tem contribuído para estratégias de autocuidado, visando respaldar a assistência prestada a esta clientela. Utilizou-se, como estratégia metodológica, a abordagem qualitativa mediante estudo descritivo. O procedimento de coleta de material envolveu a realização de entrevistas com roteiro semiestruturado realizadas com os hipertensos cadastrados no HIPERDIA. A análise foi realizada respeitando os preceitos da análise de conteúdo proposta por Bardin. As considerações dos sujeitos demonstram que a Consulta de Enfermagem têm possibilitado, por meio da educação em saúde, o acesso ao conhecimento acerca da patologia com uma postura de apoiador, motivando os clientes na conscientização de hábitos saudáveis, uma vez que, para os usuários entrevistados, as alterações no estilo de vida não envolvem procedimentos simples. Vê-se a necessidade de divulgação do trabalho realizado pelo enfermeiro durante a Consulta de Enfermagem, ao hipertenso ou não, para o fortalecimento de sua atuação e do reconhecimento da identidade profissional do enfermeiro junto à população.

Palavras-chave: Enfermagem de atenção primária. Hipertensão. Relações enfermeiro-paciente.

\section{CONSIDERATIONS FOR CONSULTATION OF NURSING: THE LOOK OF HIPERTENSIVE ATTENTION PUBLIC PRIMARY IN THE MUNICIPALITY OF PETROLINA / PE}

\begin{abstract}
The aim of this study was to know the understanding that hypertensives have about nursing consultation held by professionals and how it has contributed to self-care strategies in order support the care provided to this customer. It was used as a methodological strategy, the qualitative approach through descriptive study. The procedure of sample collection involved conducting semi-structured interviews conducted with hypertensive registered in HIPERDIA. The analysis was carried out respecting the precepts of content analysis proposed by Bardin. The considerations of the subjects showed that the nursing consultation have enabled through health education, access to knowledge about the disease with a posture supporter, motivating clients in awareness of healthy habits, since, for the users interviewed, changes in lifestyle does not involve simple procedures. Sees the need to extend the work done by nurses during the nursing consultation, the hypertensive or not, to strengthen their role and recognition of the nurse's professional identity among the population.
\end{abstract}

Keywords: Primary Care Nursing. Hypertension. Nurse-patient relations 


\section{CONSIDERAÇÕES INTRODUTÓRIAS}

No Brasil, a atenção primária à saúde tem caráter estratégico na realização de ações de promoção, vigilância em saúde, prevenção, assistência e acompanhamento longitudinal dos portadores de doenças crônicas não transmissíveis, sendo responsável pelo encaminhamento precoce dos pacientes para a atenção especializada de média complexidade, onde será instituída a terapêutica apropriada para retardar a progressão da patologia, prevenir suas complicações e garantir um preparo adequado para a iniciação às terapias de acompanhamento (PEIXOTO; et al., 2013).

Para esta pesquisa considerar-se-á as doenças crônicas não transmissíveis conceituadas como afecções que acometem o indivíduo por um longo período de tempo podendo apresentar períodos de exacerbação ou de melhora sensível. Dentre elas, as doenças cardiovasculares têm sido citadas como as mais comuns, destacando-se a Hipertensão Arterial Sistêmica (HAS) (OLIVEIRACAMPOS; et al., 2013).

A Hipertensão Arterial Sistêmica pode ser definida como uma síndrome em que há aumento na tensão arterial, associada a desequilíbrios metabólicos, hormonais e alterações tróficas, uma vez que existe uma variação contínua da pressão arterial entre hipertensos e essa variabilidade apresenta maior amplitude e está relacionada a um prognóstico insatisfatório (SOCIEDADE BRASILEIRA DE CARDIOLOGIA, HIPERTENSÃO E NEFROLOGIA, 2011).

A atenção primária à saúde tem a estratégia Saúde da Família como porta de entrada para acompanhamento, prevenção e promoção à saúde, principalmente dos indivíduos acometidos por doenças crônicodegenerativas. Giovanella (2008) corrobora com o exposto, ao citar que a Estratégia Saúde da Família está centrada na promoção da saúde e na intervenção precoce dos fatores que a predispõem ao risco, logo, possibilita a identificação e acompanhamento, de forma mais eficaz, dos portadores de HAS.

Em uma equipe de Saúde da Família, o enfermeiro faz parte do grupo de profissionais mínimos exigidos e sua atuação é crucial no acompanhamento dos usuários. Dentre as atividades desempenhadas por ele, destaca-se a Consulta de Enfermagem, lócus de encontro entre enfermeiro/cliente com identificação dos problemas envolvendo o processo saúdedoença, realizadas prescrições e implementações de enfermagem que colaboram para promoção, proteção, recuperação ou reabilitação do cliente com amparo legal mediante a Lei do Exercício Profissional da Enfermagem de $n^{\circ} 7498 / 86$ (BORGES; PINHEIRO; SOUZA, 2012).

Uma vez que a consulta de enfermagem é considerada uma importante estratégia no acompanhamento a esses clientes, torna-se, então, necessário conhecer de que forma esses 
indivíduos entendem a consulta e como ela tem contribuído para estratégias de autocuidado, visando respaldar a assistência de enfermagem prestada a esta clientela.

\section{ASPECTOS METODOLÓGICOS}

Trata-se de um estudo descritivo com abordagem predominantemente qualitativa, uma vez que se aplica ao estudo da história, das relações, das representações, das crenças, das percepções e das opiniões, consequência das interpretações que os indivíduos fazem a respeito de como vivem, constroem seus artefatos e a si mesmos, sentem e pensam (MINAYO, 2007, p.57).

Neste sentido, foi possível contar com a colaboração dos gestores do município de Petrolina/PE e da enfermeira da Estratégia Saúde da Família que, gentilmente, permitiu o acesso dos pesquisadores aos usuários portadores de Hipertensão Arterial Sistêmica e cadastrados no HIPERDIA (Sistema de cadastramento e acompanhamento de hipertensos e diabéticos captados no Plano Nacional de Reorganização da Atenção à hipertensão e diabetes gerando informações para gerentes locais, gestores das secretarias de municipais, estaduais e Ministério da Saúde) (GOMES; SILVA; SANTOS, 2010).

A pesquisa foi realizada individualmente, mediante visitas domiciliares, uma vez que a unidade de saúde não dispunha de espaço físico adequado que possibilitasse o procedimento de coleta de dados, e observado os aspectos éticos preconizados pela Resolução 196/96 do Conselho Nacional de Saúde que norteia a pesquisa com seres humanos (Revogado pela Resolução $\mathrm{n}^{\circ}$ 466/2012).

$\mathrm{O}$ projeto foi encaminhado à Secretaria Municipal de Saúde, no setor da Atenção Primária, para fornecer informações e obtenção da concessão da devida autorização, em seguida foi enviado à análise, sendo aprovado pelo Comitê de Ética do instituto de Medicina Integral Professor Fernando Nogueira (IMIP) com CAAE: 0163.0.099.000-09 e protocolo $\mathrm{n}^{\mathrm{o}}$ 1555-09. A partir da exposição dos princípios éticos, os sujeitos que concordaram em participar da pesquisa assinaram o termo de consentimento livre e esclarecido.

Para atender aos objetivos propostos na coleta de dados foi utilizada a entrevista com roteiro semiestruturado gravadas em aparelho áudio. Após contínuas escutas, as gravações foram transcritas na íntegra, de modo a não se perderem aspectos importantes para a interpretação e análise.

Os dados foram tratados conforme o método de Análise de Conteúdo que, segundo Bardin (2011) representa um conjunto de técnicas de análise das comunicações e seu objetivo consiste na inferência de conhecimentos relativos às condições de produção que recorre a indicadores (quantitativos ou não). Essa análise foi constituída por três etapas: 
pré-análise, exploração do material com tratamento dos resultados obtidos e interpretação.

A pré-análise foi constituída pela escolha do objeto de estudo, bem como a formulação dos objetivos do trabalho, necessário para proceder à constituição do "corpus". O corpus constitui o conjunto do material que será submetido à análise, no caso, a leitura flutuante das transcrições dos depoimentos dos hipertensos entrevistados.

Outra etapa didaticamente descrita por Bardin (2011) é a exploração do material que consiste em uma fase longa com procedimentos de codificação ou enumeração em função de regras previamente formuladas. A codificação consiste na transformação que ocorre, segundo regras precisas em relação aos dados brutos, do texto analisado. No contexto estudado, foi possível codificar fragmentos dos depoimentos dos hipertensos em unidades de representações daquilo que descreviam acerca das consultas de enfermagem realizadas pelos profissionais de enfermagem.

Após a escolha das unidades de análise foi realizada a seleção das categorias pelo reagrupamento dos temas específicos da realização laboral dos profissionais durante a consulta de enfermagem aos hipertensos: a) A consulta de enfermagem é...; b) Contribuições da consulta de enfermagem; c) Mudanças que promovem o autocuidado.
A interpretação dos resultados constituiu a terceira etapa da proposta metodológica quando houve o confronto entre os depoimentos dos entrevistados e a revisão de literatura nacionalmente divulgada quando foi possível elucidar as limitações e potencialidades das consultas de enfermagem prestadas aos hipertensos acompanhados em uma unidade de saúde da família do município de Petrolina/PE.

\section{DISCUSSÃO DOS RESULTADOS}

Os depoimentos relacionados ao entendimento dos entrevistados quanto à Consulta de Enfermagem, em sua maioria, caracterizaram-na como uma atividade realizada pela enfermeira, cujos objetivos se resumem em realizar orientações e fazer questionamentos sobre o uso dos medicamentos e mudanças nos hábitos de vida, conforme os fragmentos a seguir:

Pra mim, assim, é a consulta que ela orienta direitinho, vê se a gente segue o tratamento corretamente, é uma consulta de acompanhamento do hipertenso (suj. 04).

É a consulta que a enfermeira pergunta se está tudo bem com a gente, ela pergunta da alimentação, se toma o medicamento direitinho, se a pessoa está sentindo alguma coisa (suj. 15). 
É a consulta que a enfermeira faz o acompanhamento para saber como a gente está se cuidando e se a pressão está ou não controlada (suj. 10).

Desse modo, percebe-se que a consulta de enfermagem é vista como uma atividade simples, com um menor nível de complexidade na atenção, uma vez que não foi considerada pelos entrevistados a capacidade do enfermeiro em avaliar a situação do processo saúde-doença, assim como, intervir para manutenção da saúde e prevenção dos agravos.

Embora o levantamento das necessidades básicas dos clientes e o fornecimento de orientações sejam aspectos inerentes e indispensáveis à assistência de enfermagem, a consulta não se limita apenas a estas etapas, a metodologia abrange ações como: levantamento do histórico de enfermagem (entrevista e exame físico), diagnóstico, planejamento de enfermagem, implementação, evolução e avaliação sendo que, na consulta ao hipertenso na atenção primária, o profissional de enfermagem solicita e avalia determinados exames, transcreve medicações e realiza o encaminhamento médico, quando necessário (BORGES; PINHEIRO; SOUZA, 2012).

De acordo com Santos et al. (2008) a Enfermagem, apesar de ser uma profissão dotada de corpo científico próprio, pautada em ensino, pesquisa e extensão e de ter apresentado inúmeros avanços científicos e tecnológicos ao longo de sua existência, ainda não é valorizada pela população, e vários são os aspectos que interferem nesse processo.

Felipe et al. (2011) constataram o desconhecimento da população sobre as atribuições e competências do profissional de enfermagem, sendo considerado um mero auxiliar do médico. A forma como o trabalho realizado pelo profissional de enfermagem é vista traz uma bagagem cultural, originada a partir do senso comum e de informações levantadas pelo pensamento popular e da comunicação social.

Para entender como a consulta de enfermagem contribui para estratégias de autocuidado faz-se necessário entender algumas perspectivas que envolvem as estratégias de autocuidado do indivíduo.

Na perspectiva de Santos et al. (2008) o uso do medicamento diariamente e as modificações nos hábitos de vida envolvem alterações na forma de viver e na própria ideia de saúde e de qualidade de vida que o indivíduo possui, logo, a sensibilização e a educação do cliente se fazem necessárias para um adequado controle da doença, possibilitando melhor convívio e tornando o indivíduo capaz de prevenir e detectar situações de risco, porém, é necessário que o educador em saúde auxilie pacientemente o cliente nas suas descobertas e reflexões sobre a realidade. 
Para Moura et al. (2011) a promoção do autocuidado e da adesão ao tratamento deve ser um dos principais objetivos a serem implementados pela Estratégia Saúde da Família. Tal desenho organizativo atua como porta de entrada e propõe um novo modelo de atencão à saúde baseado nas ações de promoção, prevenção, proteção, recuperação e reabilitação. Logo, o profissional de saúde age apenas como um facilitador ao utilizar técnicas ou meios que levem o cliente a realizar práticas de autocuidado.

Neste estudo, foi verificado que a educação em saúde desenvolvida durante as Consultas de Enfermagem estimularam hábitos de autocuidado salutares, conforme os trechos a seguir:

Quando eu comecei fazer a caminhada que ela falou, eu acho que a pressão ficou mais controlada (suj 01).

Antes né? eu não me cuidava tanto, mas agora que eu passei a escutar mais a enfermeira em algumas coisas. É muita coisa, mas, mesmo assim, eu faço o que posso e acho que estou melhor (suj 07).

Eu me cuido, já modifiquei a alimentação aqui em casa, faço a caminhada e tomo os medicamentos todos os dias, nunca fumei e nem bebo, por isso que minha pressão é controladinha (suj. 08).
Eu estou bem agora, procurei me cuidar, né? Fazer o que ela diz, diminuir sal e gordura, só não faço caminhada porque não posso (suj 10).

Além do atendimento às recomendações feitas pela enfermeira na consulta, pôde-se perceber nas falas acima, o reconhecimento pelos sujeitos do melhor controle da doença e de uma sensação de bem estar após terem realizado o que foi proposto nas orientações, revelando, assim, a contribuição da Consulta de Enfermagem na manutenção da saúde e na prevenção de agravos.

Moura et al. (2011) colocam que o enfermeiro, no seu papel educador e transformador, admite as particularidades do cliente, favorecendo a aquisição de habilidades necessárias para o autocuidado e contribuindo significativamente para a promoção e restauração da saúde, como também, para conscientização na qualidade de vida.

A fim de tornar efetiva a relação com o cliente, o enfermeiro deve comprometer-se com o mesmo, interessando-se por ele, por seus pensamentos, sua vida, seus anseios e estar disposto a ajudá-lo a buscar respostas ou saídas para situações possíveis aos reparos, como também, tornar-se capaz de apoiá-lo naqueles momentos que não podem ser modificados (FELIPE; ABREU; MOREIRA, 2008).

O contato entre o profissional de saúde e o portador de HAS deve ser constante, uma vez 
que se trata de uma patologia crônica e o indivíduo necessita de acompanhamento para o controle da doença, logo, é importante o estabelecimento de vínculo entre profissional e cliente o que, por sua vez, dependerá da relação interpessoal que foi construída.

Neste estudo foi possível verificar que os hipertensos entendem a Consulta de Enfermagem como uma atividade na qual a enfermeira consegue estabelecer uma interação humanística na assistência, os clientes se sentem valorizados e importantes durante o atendimento, conforme os trechos abaixo:

A enfermeira dá atenção à gente na consulta, pára para nos escutar, aconselha e tem interesse por a gente (suj. 16).

Eu gosto da consulta, a enfermeira é muito paciente, sabe? Ela escuta tudo que você tem para falar, a consulta dela é boa por isso (suj 14).

Ela é boazinha, atende direitinho e sempre resolve os problemas quando eu peço, é atenciosa, eu me entendo muito bem com ela (suj 01).

Se eu precisar de alguma coisa eu falo e, aí, ela tenta resolver (suj. 10).

Nas falas acima, fica evidenciado que os hipertensos encontram na Consulta de
Enfermagem, devido ao diálogo existente, um momento de escuta, além de ficar claro a disposição da enfermeira em buscar a resolutividade do problema relatado pelo cliente, ou seja, pode-se dizer que a mesma exerce, durante a consulta, o acolhimento de forma humanizada.

De acordo com Rover et al (2011) acolher significa receber bem, ouvir as necessidades do indivíduo, procurar formas de entendê-las e solidarizar-se com elas, além de elaborar formas adequadas de receber os diferentes modos como os clientes procuram ajuda nos serviços de saúde. Para Mitre, Andrade, Cotta (2013) o acolhimento expressado como um ato ou efeito de acolher significa, em seus vários conceitos, uma ação de aproximação, um "estar com" e "perto de", dito de outra forma, uma maneira de favorecer a inclusão.

Ainda sobre o acolhimento e humanização houve, por parte dos hipertensos, a iniciativa de comparar a Consulta de Enfermagem com aquela realizada pelo profissional médico, conforme verificado nas falas abaixo:

Ela pergunta mais que os próprios médicos, por isso que gosto da consulta dela, ela atende direitinho, escuta tudinho e, aí, ela ajuda como pode, né? (suj 07).

Eu acho que ela atende bem, tem paciência, diferente de muitos médicos que têm por aí, sabe? Ela não fica apressada na consulta (suj 18). 
No estudo desenvolvido por com hipertensos e diabéticos também foi verificado a mesma opinião, de que a Consulta de Enfermagem é diferente da Consulta Médica, pois proporciona o estabelecimento de uma relação mais próxima e individual com os clientes, marcada pela informalidade e flexibilidade (FELIPE; ABREU; MOREIRA, 2008).

O médico, em particular, por um desvio de formação, está muito pouco treinado para dispender tempo com seu paciente, passandolhe informações detalhadas sobre hábitos de vida. A linguagem que utiliza e a própria postura que adota de distanciamento são outras barreiras para a comunicação.

A Consulta de Enfermagem, ao considerar a humanização, colabora para o estabelecimento de relações interpessoais mais dedicadas, menos desiguais e menos autoritárias, por utilizar estratégias que ultrapassam a superficialidade de um atendimento biológico. No atendimento humanizado percebe-se que, além da atenção dispensada à doença, o hipertenso é acolhido como pessoa e, nesse contato, há um envolvimento entre ele e quem realiza a consulta (MOURA et al, 2011).

Quando indagados acerca de sugestões para elevar a qualidade da assistência na Consulta de Enfermagem, os sujeitos relataram que a prescrição de medicações é o item que falta para a melhoria do atendimento, conforme é verificado nos depoimentos em tela:
Ah, se ela passasse remédio era melhor, mas ela já me explicou que não pode, então, o resto está bom, precisa mudar nada não (suj. 04).

O bom, assim, era se ela passasse remédio, para a gente não ficar dependendo do médico, né? Mas eu sei que ela não pode porque o trabalho dela é de enfermeira chefe, né?(suj 16).

A consulta dela é boa, mas se pelo menos ela pudesse passar remédio era melhor (suj 19). Através das falas dos sujeitos, verifica-se que os mesmos estão satisfeitos com a consulta feita pela enfermeira, porém, gostariam que ela realizasse uma prática que não é de sua competência. De acordo com a Lei do exercício profissional de $\mathrm{N}^{\circ}$. 7498/86, o enfermeiro poderá prescrever apenas medicamentos estabelecidos em programas de saúde pública e em rotina aprovada pela instituição de saúde.

No Caderno de Atenção Primária sobre Hipertensão Arterial, é colocado que no programa de controle da doença, a enfermeira só esta autorizada a transcrever as medicações daqueles clientes cuja pressão arterial está controlada e que não apresentaram intercorrências, sendo atribuição do profissional médico a indicação da terapia medicamentosa, uma vez que sua formação o habilita para tal prática (BRASIL, 2010). 
Pode-se ainda inferir que, embora os sujeitos reconheçam a habilitação da profissão ao citarem que a prescrição de medicações não cabe à enfermeira, desconhecem os reais objetivos da Consulta de Enfermagem e de que sua abordagem e metodologia são e devem permanecer diferentes da consulta médica, uma vez que cada área possui suas especificidades, mesmo que haja entre elas objetivos comuns.

\section{CONSIDERAÇÕES FINAIS}

A Consulta de Enfermagem é uma atividade de importância significativa, especialmente nos grupos que se encontram em situações de risco e que precisam de uma atenção mais direcionada e constante, como é o caso dos portadores de HAS.

Este estudo possibilitou verificar a ótica dos hipertensos em relação à Consulta de Enfermagem e os fatores relacionados a ela para que, através da avaliação feita pelo cliente, os enfermeiros possam repensar suas práticas no intuito de melhorar a qualidade do seu atendimento.

As considerações dos sujeitos demonstram que a Consulta de Enfermagem têm possibilitado, por meio da educação em saúde, o acesso ao conhecimento acerca da patologia com uma postura de apoiador, motivando os clientes na conscientização de hábitos saudáveis, uma vez que, para os usuários entrevistados, as alterações no estilo de vida não envolvem procedimentos simples. Os sujeitos referem que estão satisfeitos com a Consulta de Enfermagem, no entanto, o desconhecimento das diferenças entre os objetivos do trabalho da enfermeira e do papel desempenhado pelo médico os levam a sugerir que o enfermeiro realize uma ação para o qual não foi habilitado, sendo compreensível, uma vez que as informações sobre os diferentes papéis dos profissionais de saúde são pouco divulgadas.

Em síntese, foi possível elucidar que os hipertensos entrevistados gostariam de ser atendidos por um profissional que tivesse uma postura acolhedora, humanística e de educador, como o enfermeiro, mas caracteriza a consulta de enfermagem de maneira simplória sem expor a complexidade e importância que a mesma oferece. Na verdade espera, do profissional da enfermagem, a autorização para prescrever medicações, sem atentar que a atenção à saúde deve ser feita de forma interdisciplinar e multiprofissional o que, de forma geral, ainda não acontece na atenção primária.

Por fim, vê-se a necessidade da divulgação do trabalho realizado pelo enfermeiro na Consulta de Enfermagem, ao hipertenso ou não, para o fortalecimento de sua atuação e do reconhecimento da identidade profissional desta categoria junto à população. 


\section{REFERÊNCIAS}

BARDIN, Laurence. Análise de Conteúdo. Lisboa: Edições 70, 2011. 393p.

BORGES, José Wicto Pereira; PINHEIRO, Nádia Marques Gadelha; SOUZA, Ana Célia Caetano de. Hipertensão comunicada e hipertensão compreendida: saberes e práticas de enfermagem em um Programa de Saúde da Família de Fortaleza, Ceará. Ciênc. saúde coletiva [online], v.17, n.1, p. 179-189. 2012.

BRASIL. Lei $n^{\circ}$. 7.498, de 25 de junho de 1986. Dispõe sobre a regulamentação do exercício da enfermagem e dá outras providências. Diário Oficial [da] República Federativa do Brasil. Brasília, DF: Ministério da Saúde, 1986. Disponível em: http://www2.camara.gov.br/internet/legislaca o/legin.htm. Acesso em: 02 jul. 2013.

- Ministério da Saúde. Resolução 196/96 do Conselho Nacional de Saúde. Dispõe sobre pesquisas envolvendo seres humanos. Brasília: Ministério da Saúde, 1996. Disponível

em: http://conselho.saude.gov.br/comissao/conep/r esolucao.html. Acesso em: 20 de janeiro de 2013.

Ministério da Saúde. Secretaria de Atenção a Saúde. Política Nacional de Humanização. Brasília, DF: Ministério da Saúde, 2010, 256 p.

. Ministério da Saúde. Resolução 466, de 12 de dezembro de 2012, do Conselho Nacional de Saúde, dispõe sobre respeito pela dignidade humana e pela especial proteção devida aos participantes das pesquisas científicas envolvendo seres humanos. Brasília: Ministério da Saúde, 2012. Disponível em: http://conselho.saude.gov.br/resolucoes/2012/ Reso466.pdf. Acesso em: 10 de julho de 2013.

FELIPE, Gilvan Ferreira et al. Consulta de enfermagem ao usuário hipertenso acompanhado na atenção básica. Rev RENE, v.12, n. 2, p.287-294, abr-mai. 2011.

FELIPE, Gilvan Ferreira; ABREU, Rita Neuma Dantas Cavalcante de; MOREIRA, Thereza Maria Magalhães. Aspectos contemplados na consulta de enfermagem ao paciente com hipertensão atendido no programa saúde da família. Rev. Esc. Enferm. USP [online], v.42, n.4, p. 620-627. 2008.

GIOVANELLA, Ligia. Atenção Primária à Saúde seletiva ou abrangente?. Cad. Saúde Pública [online], v.24, suppl.1, p. 21-23. 2008.

GOMES, Tiago José de Oliveira; SILVA, Monique Vércia Rocha e; SANTOS, Almira Alves dos. Controle da pressão arterial em pacientes atendidos pelo programa Hiperdia em uma Unidade de Saúde da Família. Rev Bras Hipertens,v.17, n.3, p.132-139, jul-set. 2010.

MITRE, Sandra Minardi; ANDRADE, Eli Iola Gurgel; COTTA, Rosângela Minardi. O acolhimento e as transformações na práxis da reabilitação: um estudo dos Centros de Referência em Reabilitação da Rede do Sistema Único de Saúde em Belo Horizonte, MG, Brasil. Ciênc. saúde coletiva, v.18, n.7, p. 1893-1902. 2013.

MOURA, Denizielle de Jesus Moreira et al. Cuidado de enfermagem ao cliente com hipertensão: uma revisão bibliográfica. Rev. bras. enferm. [online], v.64, n.4, p. 759-765. 2011.

OLIVEIRA-CAMPOS, Maryane et al. Impacto dos fatores de risco para doenças crônicas não transmissíveis na qualidade de vida. Ciênc. saúde coletiva [online], v.18, n.3, p. 873-882. . 2013.

PEIXOTO, Eline Rezende de Morais et al. Diálise planejada e a utilização regular da atenção primária à saúde entre os pacientes diabéticos do Município de Belo Horizonte, 
Minas Gerais, Brasil. Cad. Saúde Pública [online], v.29, n.6, p. 1241-1250. 2013.

ROVER, Marina Raijche Mattozo et al. Humanizando o acesso de medicamentos na farmácia escola UFSC/PMF. Rev Universidade Vale do Rio verde, Três Corações, v. 9, n. 3, suplemento. 2011.

SANTOS, Sueli Maria dos Reis et al. A consulta de enfermagem no contexto da atenção básica de saúde, Juiz de Fora, Minas Gerais. Texto contexto - enferm. [online], v.17, n.1, p. 124-130. 2008.

SOCIEDADES BRASILEIRAS DE CARDIOLOGIA, HIPERTENSAO E NEFROLOGIA.V. Diretrizes de Monitoração Ambulatorial Da Pressão Arterial (MAPA) e III Diretrizes de Monitoração Residencial da Pressão Arterial (MRPA). Arq. Bras. Cardiol [online], v.97, n.3, suppl.3, p. 1-2. 2011. 